\title{
LA BIOÉTICA Y EL AUTOCUIDADO DE LA SALUD: IMPERATIVOS PARA LA FORMACIÓN INTEGRAL EN LA UNIVERSIDAD
}

\author{
Zoila Rosa Franco Peláez ${ }^{1}$ Jorge Alberto Duque Escobar ${ }^{1}$
}

Resumen: El autocuidado es responsabilidad del ser humano. La formación integral (FI), como proceso de desarrollo humano, ubica al docente universitario en la socialización terciaria de los estudiantes, reconociéndolos como seres dignos y sujetos de derechos y deberes. La FI fomenta un diálogo multidisciplinario y pluralista sobre cuestiones éticas entre todas las partes interesadas, incluyendo la sociedad en su conjunto. Objetivos: identificar significados que los docentes atribuyen al autocuidado en la FI, como deber bioético, y desentrañar motivos de los docentes para incluir el autocuidado en la FI de los estudiantes. Métodos: investigación cualitativa, de corte comprensivo con grupo focal; unidad de trabajo: docentes; unidad de análisis: expresiones de los participantes. Conclusiones: para asumir el compromiso bioético, es necesario el autocuidado en la FI, mediante estrategias concienciadoras en la cotidianidad, capaces de transformar el actuar de los universitarios.

Palabras clave: bioética, autocuidado, formación integral, educación superior

\section{Bioethics and auto health care: imperatives for integral training in the university}

\begin{abstract}
Self care is the responsibility of human beings. Integral training (IT) as a process of human development places university professors as tertiary socialization for students, recognizing them as beings with dignity, subjects of rights and duties. IT promotes multidisciplinary and pluralist dialogue about bioethics topics among all interested parties, including society as a whole. Objectives: To identify meanings of auto health care attributed by professors in IT as bioethical duty and to unfold professors motives to include auto health care in IT for students. Methods: qualitative research of comprehensive nature by focus group discussion; unit of study: professors; unit of analysis: statements of participants. Conclusions: in order to compromise with bioethics, auto health care is needed in IT by taking conscientious strategies in the daily life, able to transform university students acting.
\end{abstract}

Key words: bioethics, auto health care, integral training, superior education

A bioética e o autocuidado em saúde: imperativos para a formaçáo integral na universidade

Resumo: O autocuidado é responsabilidade do ser humano. A formação integral (FI) como processo de Desenvolvimento Humano situa o docente universitario na socialização terciária dos estudantes, reconhecendo-os como seres dignos, sujeitos de direitos e deveres, para fomentar um diálogo multidisciplinar e pluralista sobre as questóes de bioética para todas as partes interessadas, incluindo a sociedade em seu conjunto. Objetivos: Identificar significados que atribuem docentes ao autocuidado na FI, como dever bioético e compreender os motivos dos docentes para incluir o autocuidado na FI dos estudantes. Métodos: investigação qualitativa, de corte compreensivo com grupo focal; unidade de trabalho: docentes; unidade de análise: expressóes dos participantes. Conclusóes: Para assumir o compromisso bioético é necessário o autocuidado na FI, mediante estratégias conscientizadoras na cotidianidade, capazes de transformar a atuação dos universitários.

Palavras-chave: bioética, autocuidado, formação integral, educação superior

\footnotetext{
${ }^{1}$ Universidad de Caldas, Manizales, Colombia

Correspondencia: zoila.franco@ucaldas.edu.co
} 


\section{Introducción}

Para introducirnos en los elementos teóricos de la investigación, examinemos una definición de bioética, consignada en la primera edición de la "Enciclopedia de Bioética" de Warren Reich. Ella hace mención a "una conducta que es examinada a la luz de los principios y valores morales", afirmación que generó mucha polémica y crítica y obligó al autor a revisarla en la segunda edición, en 1995: la bioética, según esta revisión, es el estudio sistemático de las dimensiones morales -incluidas la visión axiológica, las decisiones, la conducta, las líneas de acción y políticas- de las ciencias de la vida y los cuidados sanitarios, con el empleo de una variedad de metodologías éticas, en un planteamiento interdisciplinar(1).

En el artículo $5^{\circ}$ de la Declaración Universal sobre Bioética y Derechos Humanos, promulgada por la UNESCO en 2005, se menciona la autonomía y la responsabilidad, lo que implica respetar las libertades fundamentales de la persona, en su facultad de adoptar decisiones, asumiendo la responsabilidad del individuo y respetando la autonomía de los demás(2).

Los paradigmas médicos surgidos desde la época del Renacimiento se redujeron al estudio de la enfermedad como proceso patológico, en un cuerpo objetivado y despersonalizado; en consecuencia, la relación médico-paciente se deshumanizó, con lamentables consecuencias que se hicieron evidentes hasta la segunda mitad de siglo pasado. Esto llevó a aceptar con rapidez el concepto de bioética, para aplicarlo especialmente en el quehacer profesional, con el surgimiento de una nueva mentalidad o, si se quiere, paradigma antropológico en esencia y antropocéntrico en forma. Sin duda, este giro parece ser mas ajustado a la dignidad de los seres humanos y a la calidad de los actos médicos y de los otros profesionales de la salud que atienden a los enfermos. Introduce también el asunto de los derechos humanos y, entre ellos, la obligación del autocuidado, que deberá ser materia importante en la formación integral de las futuras generaciones(3).

Reconocer la dignidad humana es reconocer el valor intrínseco de cada ser humano, lo que implica respeto, solidaridad y libertad, como ele- mentos substanciales de lo que Adela Cortina llama, "crecer en humanidad": "La razón de la dignidad pone de relieve el tema kantiano de la preponderancia de las emociones racionales, que ofrece el criterio sobre la naturaleza de tal axioma: dignidad es explicitar siempre la condición de ser racional, lo que denota la decencia que significa no encubrir esa condición con actos de acuerdo, porque tienen criterios que las subordinan y degradan. Por tanto, si el ser humano asume esa decencia es en primera instancia ante él mismo una persona merecedora de respeto. Si además esa decencia es exteriormente apreciada, entonces ese respeto puede ser compartido por los demás y es un garante de las relaciones entre los seres pensantes. En consecuencia, los juicios dignidad y respeto son correlatos para Kant.

La dignidad se transforma en un derecho moral básico, que garantiza al ser racional respeto para consigo mismo y respeto para los demás. En este sentido, se habla de obligaciones mutuas. Del postulado inicial acerca del valor intrínseco de los seres humanos se derivan deberes en el trato con los congéneres. Se asume que si todos poseen un valor sustancial, deben ser reconocidos como tales por los demás. A su vez, ellos están obligados a actuar de conformidad con ese supuesto"(4).

Los planteamientos anteriores invitan a reconocer uno de los fundamentos con que se constituye la columna vertebral de la bioética, como es el reconocimiento, la aceptación y el respeto activo de la dignidad de la vida y, de manera consubstancial, la dignidad humana como premisa que robustece el tono moral. De tal manera, tal fundamento permea las elecciones, decisiones y conductas en el actuar cotidiano que entrańa la responsabilidad, entendida como conciencia de las consecuencias que, para bien o para mal, se derivan de toda las actuaciones humanas.

El segundo tema a revisar en el marco teórico de la investigación corresponde al autocuidado, entendido como la capacidad que debe desarrollar cada individuo, durante su proceso vital, en aras de promover, proteger y mantener la vida y la salud para lograr una buena calidad de vida, en el cumplimiento de un proyecto de desarrollo humano armónico. 
El autocuidado de la vida y la salud implica autodeterminación, señalando la responsabilidad que asume el joven cuando, en el medio universitario, se enfrenta a una diversidad de experiencias culturales y generacionales que le obligan a elegir cursos de acción relacionados con factores protectores o de riesgo para su salud.

Dentro de la formación universitaria es imperativo asumir la bioética como una perspectiva para el autocuidado, lo que implica informar a los estudiantes sobre sus postulados, principios y valores, de manera que, una vez interiorizados e incorporados en sus rutinas cotidianas, transformen comportamientos de riesgo para la vida y la salud en conductas protectoras.

Atendiendo a lo anterior, las universidades tienen la responsabilidad social de transformarse y de transformar las circunstancias adversas relacionadas con los determinantes sociales de la salud —incluyendo los espacios donde viven, estudian y trabajan las personas, esto es, donde trancurre su proceso vital(5)—, integrando procesos orgánicos y psicológicos, y relaciones personales y sociales necesarios para el funcionamiento adecuado y el disfrute de una vida digna. La universidad, a través de sus docentes y estudiantes, debe convertirse en un modelo de autocuidado.

Heidegger alude al cuidado como forma de ser esencialmente ética: algo más que un acto y una actitud. Plantea el autor que el cuidado es existenciariamente a priori a toda posición y conducta fáctica del ser ubicado, es decir, que se halla siempre en ella. El cuidado se encuentra en la raíz primera del ser humano, antes de que este actúe, y todo cuanto haga irá siempre acompañado e impregnado de cuidado. Significa reconocer que el cuidado es un modo de ser propio, es una dimensión frontal, originaria, ontológica, imposible de desvirtuar(6). En este orden de ideas, el autocuidado — cuidado referido a sí mismo, como responsabilidad de proporcionarse una vida saludable- está centrado en cada persona antes de su formación. Siguiendo una de las recomendaciones de la Carta de Ottawa (1986): "las personas podrán alcanzar su plena salud potencial cuando sean capaces de asumir el control de todo lo que determine su estado de salud; esto se aplica igualmente a hombres y mujeres"(7).
Por otro lado, corrientes filosóficas como el estoicismo generaron desarrollos importantes en torno al autocuidado como práctica, llamado por ellos "cultivo de sî", lo cual valora la importancia de las relaciones del individuo consigo mismo(8). Para los griegos, este principio dominaba el arte de la existencia, hasta el punto de ser la diferencia fundamental del hombre con otros seres vivos. Como afirma Epicteto, el ser humano ha sido confiado a la inquietud de sí y es ahí donde reside su diferencia fundamental con otros seres vivos. Para él la inquietud de sí era un privilegio-deber, un don-obligación que nos asegura la libertad, impulsándonos a tomarnos como sujetos ${ }^{2}$ de toda aplicación(8).

Respecto del concepto de "formación integral", presentamos el desarrollado por Luis Enrique Orozco, porque recoge elementos que se acercan al ejercicio de la responsabilidad, bajo el imperativo bioético de las universidades saludables:

"La formación integral va más allá de la capacitación profesional, aunque la incluye. Es un enfoque o forma de educar. La educación que brinda la universidad es integral en la medida en que enfoque a la persona del estudiante como una totalidad y que no lo considere únicamente en su potencial cognoscitivo o en su capacidad para el quehacer técnico o profesional. El ámbito de la formación integral es el de una práctica educativa centrada en la persona humana y orientada a cualificar su socialización, para que el estudiante pueda desarrollar su capacidad de servirse en forma autónoma del potencial de su espíritu, en el marco de la sociedad en que vive, y pueda comprometerse con sentido histórico en su transformación"(9).

Por lo tanto, existe una estrecha conexión entre bioética, autocuidado y formación integral, como fundamentos que permiten una visión unitaria del ser humano en el proceso educativo, para superar la fragmentación que aún continúa creando profundas inconsistencias en la historia humana.

\footnotetext{
${ }^{2}$ En la cita de Epicteto, los autores consideran pertinente cambiar la palabra "objeto" por la palabra "sujeto", con el fin de ser coherentes con dichos postulados, entre los cuales se encuentra el principio de inobjetabilidad, que hace referencia a lo que plantea Kant respecto de la dignidad humana: el hombre es un fin en sí mismo, más que un medio para ser utilizado(10).
} 
La constitución de lo humano es muy diversa. Edgar Morin sostiene que cada ser humano, desde su concepción, está influenciado por una serie de factores del ambiente humano, natural y del contexto social y cultural donde tuvo su origen(11). Ese rico juego de influencias internas y externas da posibilidades distintas y oportunidades particulares para el desarrollo. En ese marco histórico, convergen a su vez muchos fenómenos que permiten construirse paulatinamente, a la vez que las estructuras orgánicas crecen y se desarrollan para establecer las relaciones con el mundo externo, en el cual la persona desplegará todos sus talentos, potencialidades y capacidades, para llevar a cabo, de la manera más armónica, su proyecto vital hasta la muerte.

En definitiva, esta discusión se enmarca en la doctrina bioética del personalismo, que reconoce que las acciones morales dependen enteramente del centro de autodeterminación llamado "persona", porque implica responsabilidad, en el sentido de saber dar razón de lo que se hace de manera proyectada, organizada, puesto que la persona es autora, causa y principio. El ser humano es moral en la medida en que es racional, inteligente e intuitivo. Se denomina "persona" al sujeto de naturaleza corpóreo-espiritual, en el que radica la actuación, la unidad, la totalidad y las características que le son propias. La persona es el todo, la perfección del todo, el compuesto de materia y espíritu que la anima; lo integral que forma al ser humano y su naturaleza, diferente de las otras especies con las que convive en el universo(12).

De lo anterior se infiere que la universidad podrá contribuir a la formación humana en la medida en que, a través de sus funciones de docencia, investigación y extensión, involucre al estudiante en vivencias intelectuales, estéticas y morales que le permitan sentirse implicado y afectivamente comprometido en prácticas específicas y valores determinados. La universidad es un espacio de compromiso práctico y colectivo. Qué prácticas y valores adopte el estudiante dependerá del rostro de la institución y de la manera como esta comprenda su sentido dentro de una sociedad determinada.

Consideramos pertinente hacer visible la bioética en el proceso de formación integral en la univer- sidad, para promover la salud y prevenir la enfermedad, y concienciar sobre la importancia del autocuidado.

\section{Métodos}

De acuerdo con estos planteamientos, los investigadores se preguntaron: la responsabilidad bioética de la universidad, ¿requiere del autocuidado como elemento central de la formación integral?

Esta investigación se realizó mediante un método de investigación social cualitativa (enfoque hermenéutico, de carácter comprensivo), que permitió entender, más que describir o explicar, el fenómeno del autocuidado como elemento central de la formación integral y responsabilidad bioética. Se empleó el grupo focal como estrategia para obtener la información mediante el desarrollo de entrevistas no estructuradas. La unidad de trabajo se constituyó con docentes de planta y ocasionales, vinculados a la universidad, atendiendo a criterios de inclusión referidos al tiempo de vinculación —no menor a 5 años—, hombres y mujeres de distintos programas. Las unidades de análisis fueron textos obtenidos de la sistematización de la información.

Se aplicó el proceso de consentimiento informado a los docentes participantes en los grupos focales, quienes decidieron aparecer como anónimos, con el fin de proteger su identidad en las publicaciones que se realicen sobre los resultados obtenidos de la investigación.

\section{Hallazgos y discusión}

Los docentes participantes expresan la responsabilidad bioética asociada con sus ideas, conceptos e imaginarios sobre el tema del autocuidado, y su importancia en la formación integral (FI), cuando afirman: "Pienso que la integralidad aboca los asuntos de salud, de lúdica, de recreación, de deporte y artísticos (...)". Igualmente, la siguiente afirmación. "Practiqué deporte, no para la competencia exclusivamente, sino también para mejorar y para promover la salud fundamentalmente; me imagino que hay más dimensiones, por ejemplo, los aspectos valóricos a través de la socioafectividad y comunicación; ¿qué aspectos valóricos se pueden ir fomentando? El respeto por el orden, la tolerancia 
y la norma (...)". En este último aparte, podría inferirse la dimensión axiológica relacionada con el autocuidado como la percepción del docente sobre su crecimiento no solo corporal, sino de todas las esferas que constituyen su ser como totalidad, haciendo alusión a los valores y principios bioéticos. "Estas se complementan, no son aisladas, sino que uno se las da al estudiante y él las va interiorizando de acuerdo con el significado que tenga para él (...)". Se observa la tolerancia activa como característica de la bioética, que nutre el autocuidado en la formación integral.

"En salud, sabemos que el estilo de vida influye en la aparición de enfermedades, por tanto, hay que hacer énfasis en ello (...)". Siendo coherentes con esta afirmación, el estilo de vida que adquiere el ser humano durante su existencia constituye la esencia del autocuidado. Lo cultural se relaciona con "las concepciones, las formas de imaginar la vida, tradiciones y creencias que tiene la gente en determinada temática, para que el estudiante sepa como actuar (...)”.

Los participantes son conscientes de la importancia de reconocer el cuerpo como expresión biológica de la vida, que hace posible la relación del individuo con la sociedad, e identifican la esfera psicológica como producto de una historia que se desarrolla en la cultura en la cual se nace. El autocuidado implica una lectura de la complejidad de aspectos que conforman la biografía de los seres humanos, porque todos ellos influyen en su salud como calidad de vida. La percepción, como capacidad mental del sujeto, le permite reconocerse como ser en crecimiento y construcción en todas sus esferas. Con relación al compromiso bioético, se reconoce la responsabilidad social de la salud, la promoción de la salud y el desarrollo social para los pueblos, lo cual es un contenido esencial que comparten los diferentes sectores de la sociedad(1). Asimismo, admiten que los estilos de vida tienen una influencia directa en el proceso de enfermar, aspecto que merece destacarse en la formación en autocuidado para la salud, máxime cuando la cultura contemporánea ha influido en la aparición de nuevas concepciones y formas de imaginar la realidad, que se afincan tanto en las creencias como en las tradiciones de las personas, al momento de conducirse en lo personal y lo colectivo.
También se afirma la influencia de lo político, al incentivar la participación de todos en las decisiones que los afectan: "En lo político, el proceso de participación tiene que ver con la cultura ciudadana, porque con ella no solo convivimos con los demás, sino que nosotros, los profesores, somos un ejemplo a seguir, y nuestros estudiantes, asi no lo quieran o no lo reconozcan, nos miran y dicen: 'este es mi profesor, porque hace esto o porque no lo hace. Si somos un ejemplo a seguir, debemos tratar de ser lo mejor posible; para mi esa es una forma de ser integral con mis estudiantes y con la universidad (...)". Se desprende de esto que cada docente debe aplicar los postulados y principios éticos que le permitan practicar el autocuidado en su actuar, porque su derecho a la salud entrańa también el deber de cautelar que su imagen sea un referente digno de emular por parte de sus estudiantes.

En la actualidad se reconoce el aporte de la educación entendida como FI en la construcción de ciudadanía, para lograr un mayor nivel de crecimiento económico, reducción de las desigualdades y aporte al desarrollo humano, dado que se considera como requisito para el empoderamiento básico de las personas y el despliegue de sus capacidades. Sin embargo, esto no se refleja en la realidad, ya que la educación se ha reducido a trasmisión de conocimientos, más cercanos a la técnica y a la tecnología que a la ciencia y la sabiduría, ignorando el contexto, con respuestas inmediatistas a las exigencias de la vida laboral, sin formar espíritus autónomos y críticos(13). Uno de los participantes afirma: "Dictar los derechos humanos es obligación en medicina, sobre todo el derecho a la salud, para que el médico conozca cuál es su responsabilidad no solo como profesional, sino como ciudadano (...)".

Esta cita hace referencia al principio de justicia dentro de la bioética social, un campo de vital importancia en la promoción de los derechos humanos fundamentales, primordialmente el derecho a la vida y la salud, y el análisis de las consecuencias prácticas de la justicia en la sociedad(12). Una parte del reconocimiento del derecho a la vida y la salud es responsabilidad intransferible del individuo. Pero, asimismo, es responsabilidad de la sociedad y, en ella, de la universidad.

En este orden de ideas, puede observarse cómo 
los docentes identifican a sus estudiantes como seres que constituyen una totalidad indivisible, cuando afirman: "En el desarrollo de la afectividad, pienso que si uno como docente logra incidir un poquito en este aspecto, el proceso de aprendizaje es un poco más asertivo (...)". Vale la pena resaltar que las emociones deben ser parte constitutiva en la formación bioética y del autocuidado. En este sentido, Nussbaum se refiere a los fundamentos psicológicos de la autonomía, lo que nos induce a relacionarla con el principio bioético que la refiere, y a la justicia respecto de las condiciones institucionales y de desarrollo para sostener un respeto liberal por la igualdad humana(14). Esta lectura es coherente con los postulados de la justicia como principio bioético.

"Uno hace uso de la integralidad de esos estudiantes; uno espera que ellos sean responsables, respetuosos, participativos, que respeten la individualidad de los compañeros y del mismo docente (...). Intento hacer una aproximación de lo que enseño al contexto vital, mediato y amplio al que pertenece el estudiante".

Los docentes expresan que una universidad saludable no depende solo de la infraestructura adecuada, de sus recursos técnicos y materiales, sino, prioritariamente, de la calidad humana de quienes la integran. Esta calidad se refleja en el cuidado de todo lo que en ella se realiza, para garantizar un clima organizacional que haga eficiente su desempeño y el servicio que presta al colectivo universitario, en primera instancia, y su proyección a la sociedad en que está inmersa. Desde el punto de vista bioético, los participantes hacen referencia a la solidaridad y cooperación que se habrá de fomentar entre los seres humanos(1).

\section{Conclusiones}

Para implementar el autocuidado vale la pena asumir las siguientes premisas:

1. Es un acto de vida que permite a cada uno convertirse en sujeto de sus propias acciones, por lo tanto, es un proceso voluntario de la persona para consigo misma.

2. Es una filosofía de vida y una responsabilidad íntimamente ligada a la cotidianidad y a las experiencias vividas, y debe estar fundamen- tada en redes de apoyo, familiares y sociales.

3. Es una práctica social que implica un nivel de conocimiento básico para la elaboración de un saber que da lugar a intercambios y relaciones interindividuales(13).

La responsabilidad bioética de la universidad debe propugnar por el autocuidado como elemento central de la formación integral. Para que los actores universitarios asuman el autocuidado como una práctica cotidiana de vida y salud, es necesario incluir en su promoción las siguientes estrategias:

1. Desarrollar autoestima en los actores universitarios y generar niveles de fortalecimiento o empoderamiento como estrategias que reviertan la internalización de la impotencia, favorezcan el sentido de control personal y desarrollen habilidades de movilización individual y colectiva, para cambiar las condiciones personales y sociales en pro de la salud. Por otro lado, al potenciar la autoestima se impulsan prácticas deliberadas de autoafirmación, autovaloración, autorreconocimiento y autoexpresión de los aspectos que favorecen el desarrollo integral.

2. Involucrar en el ámbito universitario el diálogo de saberes que, como método bioético, permite identificar, interpretar y comprender la vida de las personas; luego, articular este diálogo con la lógica científica y recomponer una visión esclarecida de la enfermedad y de la salud que se traduzca en comportamientos saludables.

3. Explorar y comprender las rupturas entre conocimiento, actitudes y prácticas, y configurar propuestas de acción y capacitación que hagan viable la armonía entre cognición y comportamiento.

4. Asumir el autocuidado como una vivencia cotidiana pues, al vivir saludablemente, la promoción del autocuidado es compartir las propias vivencias. En la medida en que el personal universitario viva con bienestar, se estará en condiciones de promocionar la salud de los demás. 
5. Contextualizar el autocuidado en todos los espacios universitarios, es decir, orientarlo de acuerdo con las características de género, etnia y proyecto vital humano, reconociendo la diversidad de rasgos que posee cada ser humano dentro de la sociedad. Cada universitario/a, tiene una historia de vida, con valores, creencias, aprendizajes y motivaciones diferentes.

6. Como institución democrática, la universidad debe generar procesos participativos; la promoción del autocuidado debe incluir el compromiso activo e informado de los actores en el cuidado de su propia salud, entendida la participación como el proceso mediante el cual la comunidad asume como propio el cuidado de sí misma y del ambiente que la rodea, dirigiendo la sensibilización a aumentar el grado de control y compromiso mutuo.

7. El agente de salud al interior de la universidad debe buscar espacios de reflexión y discusión acerca de lo que la gente sabe, vive y siente sobre las diferentes situaciones de la vida y la salud, con lo cual puede identificar prácticas de autocuidado favorables, desfavorables e inocuas, y promover, mediante el proceso de FI y de reflexión-acción, un regreso a la práctica para transformarla(13).
Es necesario reconocer el autocuidado de la salud como un compromiso bioético de la formación integral. Además, adoptar estrategias concienciadoras en la vida cotidiana, capaces de generar cambios reales en el actuar de los universitarios, con el fin de crear permanentemente estilos de vida saludables.

Al hablar de formación integral, es imperativo contemplar la esfera biológica como base estructural orgánica que hace posible la expresión de la vida. La formación en autocuidado para la salud constituye la esencia de la formación humana que favorece la realización del proyecto vital, acorde con la dignidad y los derechos de los seres humanos.

La multiplicidad de roles que desempeña el ser humano, desde el proceso de socialización primaria hasta la socialización terciaria que se realiza en la universidad, entraña la responsabilidad del autocuidado como fundamento de la edificación que dará forma a un ser íntegro, entendido como una persona madura, capaz de desplegar cada una de las esferas que lo constituyen, para consolidar la realización de sus metas. 
La bioética y el autocuidado de la salud - Zoila Rosa Franco Peláez, Jorge Alberto Duque Escobar

\section{Referencias}

1. Postigo SE. Concepto de bioética: definición, método y partes. Fundamentación antropológica y ética en bioética, 2006. Disponible en: http://www.bioeticaweb.com/content/view/4335/756/

2. UNESCO. Declaración Universal sobre Bioética y Derechos Humanos. Paris; 2005.

3. Mendoza VJ. Reflexiones con mucho fundamento En: Gómez Gallego R. La dignidad humana en el proceso de salud enfermedad. Bogotá: Editorial Universidad de Rosario; 2008: 13-19.

4. Gómez Gallego R. La dignidad humana en el proceso de salud enfermedad. Bogotá: Editorial Universidad de Rosario; 2008: 46-47.

5. Damestre M. Determinantes sociales de la salud. Disponible En: http://www.atinachile.cl/content/view/18783/Determinantes-Sociales-de-la-Salud.html

6. Ferrater MJ. Diccionario de filosofía. 2a edición. Barcelona: Ariel; 2009: 762-763.

7. Carta de Otawa. Disponible en: http://www.famp.es/racs/intranet/otras_secciones/documentos/CARTA\%20DE\%20 OTTAWA.pdf

8. Foucault M. Historia de la sexualidad. La inquietud de sí. México: Siglo XXI; 1987.

9. Orozco L. La formación integral: mito o realidad. Disponible En: http://mail.ups.edu.ec/universitas/publicaciones/universitas 10/contenidospdf//laformacionintegral10.pdf.

10. Kant E. La fundamentación de la metafísica de las costumbres. 5a edición. Madrid: Espasa Calpe; 1997.

11. Morin E. La epistemología de la complejidad. Disponible en: http://www.pensamientocomplejo.com.ar/docs/files/ Morin-Edgar\%20Epistemologia\%20de\%20la\%20Complejidad.pdf

12. León Correa FJ. Temas de bioética social. Santiago de Chile: Fundación Interuniversitaria Ciencia y Vida; 2011: 46-79.

13. Uribe T. El autocuidado y su papel en la promoción de la salud. Disponible en: http://bases.bireme.br/cgi-bin/wxislind. exe/iah/online/?IsisScript=iah/iah.xis\&src=google\&base=LILACS\&lang=p\&nextAction=lnk\&exprSearch=396722\&in dexSearch=ID

14. Nussbaum MC. El ocultamiento de lo humano. Buenos aires: Kats editores; 2006: 29.

Recibido: 17 de julio de 2013

Aceptado: 29 de septiembre de 2013 